\title{
Some aspects of interactivity between endocrine and immune systems required for successful reproduction
}

\author{
Andrea Weghofer ${ }^{1,2}$, Eric Himaya ${ }^{1,3}$, Vitaly A Kushnir ${ }^{1}$, David H Barad ${ }^{1,4}$, Emanuela Lazzaroni-Tealdi ${ }^{1}$, Yao Yu ${ }^{1}$, \\ Yan-Guang $\mathrm{Wu}^{1}$ and Norbert Gleicher ${ }^{1,4^{*}}$
}

\begin{abstract}
Background: In successful reproduction, endocrine and immune systems closely interact. We here attempt to further elucidate the relationship between androgen levels, systemic activation of the immune system and reproductive success in infertile women, utilizing 2 distinct infertile patient cohorts.

Methods: In Group 1, we investigated 322 women (ages 38.6 +/- 5.4 years) at initial presentation; in Group 2125 women undergoing in vitro fertilization (169 IVF cycles, ages $38.9+$ /- 5.5 years). In Group 1, we assessed androgens and an immune panel, previously demonstrated to discriminate between activated quiescent immune systems; in Group 2, utilizing the same immune panel, we investigated whether immune system activation relates to embryo quality in IVF cycles.

Results: No individual immune test within the immune panel was associated with androgen levels. The total/free testosterone ratio (TT/FT) was, however, significantly associated with presence of gammopathies (in $\lg G$, $\lg \mathrm{M}$, $\lg \mathrm{A}$, $\left.\lg E_{;} P=0.026\right)$. Surprisingly, immune system activation was associated with significantly improved embryo quality $(P=0.008)$, a finding persistent after adjustment for age and repeat IVF cycles $(P=0.006)$.

Conclusions: Association of immune system activation with improved embryo quality concurs with previously reported immune activation in association with normal functional ovarian reserve (FOR) and normal androgen levels, while, counter intuitively, hypoandrogenism and low FOR are associated with lack of immune system activation. Mild immune system activation, therefore, likely appears essential for establishment of pregnancy, and may be regulated by androgens.
\end{abstract}

Keywords: Immune system activation, Androgens, Testosterone, In vitro fertilization (IVF), Embryo quality, infertility

\section{Background}

Since the implanting embryo is a paternal semi-allograft, successful implantation in the maternal uterus is akin to allogeneic transplantation of a "mini-multiorgan transplant" to the mother. Establishment of pregnancy is, therefore, an immunologically mediated process.

To tolerate the transplant, the mother's immune system has to "reprogram" itself from rejection to tolerance. How this happens is only incompletely understood [1], but evidence suggests that a normal immune system does

\footnotetext{
* Correspondence: ngleicher@thechr.com

${ }^{1}$ Center for Human Reproduction, New York, NY, USA

${ }^{4}$ Foundation for Reproductive Medicine, New York, NY, USA

Full list of author information is available at the end of the article
}

this well, while excessively activated immune systems (severely hyper allergenic women or women with inflammation and/or active autoimmunity), do not. Inadequate maternal tolerance then leads to allogeneic maternal immune responses against the implanting fetus and failed implantation and/or pregnancy loss [2].

Since even healthy individuals frequently, demonstrate laboratory evidence suggestive of immune system hyperactivity, normal quiescence versus activation is not well defined. For example, autoimmune abnormalities are frequently found in association with allergies [3] or even in normal patient populations $[4,5]$, yet, even if just sub-clinical, have been associated with reproductive problems, including infertility, miscarriage risk $[6,7]$ and 
complications of pregnancy, such a premature labor and preeclampsia/ eclampsia $[8,9]$.

We recently noted that women with low functional ovarian reserve (LFOR) at practically all ages are characterized by relative hypoandrogenism [10]. Yet, somewhat counter intuitively, we also discovered that they demonstrated no evidence whatsoever of immune system activation as we initially had hypothesized; yet, young, healthy control patients with normal androgen levels, to some degree did demonstrate evidence of immune system activation [11].

Based on these unexpected findings, we further hypothesized that normal androgen levels, in females approximately equally adrenal and ovarian in origin, depend on mild immune system activation. In absence of activation by an immune system - driven androgen production factor (APF), androgen levels decrease and androgen-dependent early stages of follicle maturation wither [2]. Under this hypothesis, the establishment of pregnancy, thus, would be dependent on mild immune system activation.

While this on first impression may sound like a counter intuitive proposition, various important stages of reproduction, including ovulation and implantation, have already been identified as "inflammatory" processes [12,13].

Here presented study was, therefore, designed to further explore observable effects of immune system activation, and how they may relate to reproductive success.

\section{Methods}

\section{Institutional review board}

Here presented study involved two distinct patient populations, both of which were identified and retrieved from our center's electronic research data base, where selected medical record data from presenting patients are stored in anonymized fashion for research purposes. Prior to inclusion in this research data bank, patients at our center at initial presentation sign an informed consent, which authorizes the use of their medical record data for research purposes, as long as data remain confidential and the identity of the patient remains protected. Both of these conditions are met when data are extracted from the center's electronic research database, and the study, therefore, underwent only expedited review and approval from the center's Institutional Review Board (ER12172012-01, December 18, 2012).

\section{Patient populations and main outcome measures}

This study involved 2 patient populations: Group 1 included 322 consecutive infertile women, who presented to our center for initial diagnostic evaluation at mean age of $38.6 \pm 5.4$ years. Among those 125 women (Group 2) underwent 169 IVF cycles, with some patients, thus, undergoing more than one IVF cycle. Their mean age was
$38.9 \pm 5.4$ years. Table 1 summarizes patient characteristics for both patient groups, and demonstrates that they in all characteristics were very similar.

In order to qualify for Group 1, patients had to have a complete initial data set of laboratory results in the center's electronic database, which, among immune test, included antinuclear antibody, an antiphospholipid antibody panel, including antibodies to $\beta 2$-glycoprotein, cardiolipin and phosphatidylserine in IgG, IgM and IgA isotypes, a lupus anticoagulant, an antithyroid antibody panel, including antibodies to thyroid peroxidase, thyroglobulin and thyrotropin receptor, total IgG, IgM, IgA and IgE levels, anti-adrenal antibodies (to 21-hydroxylase) and anti-ovarian antibodies. All of these laboratory tests were obtained via commercially available assays.

Because this panel of tests has in studies been proven effective in discriminating with high sensitivity, though relatively poor specificity, women with immune system activation from those with a quiescent immune system, it is part of our center's routine initial fertility evaluation at time of presentation. Fully expecting a high degree of false-positivity, any positive finding in the panel is clinically considered to represent immune system activation, and only complete absence of any positive findings is clinically considered a quiescent immune system [11].

In this context the expectation is to achieve high sensitivity but poor specificity of diagnosis. This means that the

Table 1 Baseline characteristics of Groups 1 and 2*

\begin{tabular}{|c|c|c|}
\hline & $\begin{array}{l}\text { Group } 1 \\
(\mathrm{n}=322)\end{array}$ & $\begin{array}{l}\text { Group } 2 \\
(n=125)\end{array}$ \\
\hline Age (years) & $38.6 \pm 5.4$ & $38.9 \pm 5.4$ \\
\hline $\mathrm{BMI}^{* *}\left(\mathrm{~kg} / \mathrm{m}^{2}\right)$ & $24.0 \pm 4.9$ & $24.1 \pm 4.6$ \\
\hline DHEA (ng/dL) & $469.2 \pm 289.8$ & $467.9 \pm 308.9$ \\
\hline DHEAS $(\mu \mathrm{g} / \mathrm{dL})$ & $253.5 \pm 189.0$ & $371.6 \pm 233.4$ \\
\hline Total testosterone (ng/dL) & $26.0 \pm 13.7$ & $33.7 \pm 23.5$ \\
\hline Free testosterone $(\mathrm{pg} / \mathrm{mL})$ & $2.0 \pm 1.5$ & $2.6 \pm 1.8$ \\
\hline $\mathrm{TSH}(\mu \mathrm{IU} / \mathrm{mL})$ & $1.9 \pm 1.2$ & $1.9 \pm 1.0$ \\
\hline Thyroid antibodies & $22.0 \%$ & $16.0 \%$ \\
\hline Anti-adrenal antibodies & $0.3 \%$ & $0 \%$ \\
\hline Anti-ovarian antibodies & $9.3 \%$ & $6.4 \%$ \\
\hline Antinuclear antibodies (ANA) & $13.0 \%$ & $7.2 \%$ \\
\hline Antiphopholipid antibodies (APA) & $14.6 \%$ & $14.4 \%$ \\
\hline Gammopathy & $32.0 \%$ & $36.0 \%$ \\
\hline Immune system activation ${ }^{* * *}$ & $59.0 \%$ & $59.2 \%$ \\
\hline \multicolumn{3}{|c|}{$\begin{array}{l}\text { *Values are presented as means } \pm \text { standard deviation or as percentages. } \\
\text { Patient characteristics did not differ between both groups except for DHEAS } \\
(\mathrm{P}<0.001) \text {, total testosterone }(\mathrm{P}<0.001) \text { and free testosterone }(\mathrm{P}<0.01) \text {, } \\
\text { all three the consequence of DHEA supplementation for women with LFOR } \\
\text { between baseline evaluation and IVF cycle start. For further detail, see text. } \\
\text { Percentages among antibodies represent percentages of patients with positive } \\
\text { antibody tests. } \\
\text { **BMI, body mass index. } \\
\text { ***Defined by presence of at least } 1 \text { positive laboratory test. }\end{array}$} \\
\hline
\end{tabular}


prevalence of immune system activation will be overestimated; yet, patients considered quiescent will, indeed, with high certainty lack immune system hyperactivity. In this context, statistical associations with immune system activation will, therefore, be clinically diluted by the presence of false positive patients and, therefore, underestimated. So demonstrated statistically significant associations, therefore, have to be considered statistically sound.

Since women with LFOR demonstrate low androgen levels [10], we now also routinely assess androgens in newly presenting patients. Group 1 patients, therefore, also had to have a completed set of androgen values in the center's research database, including dehydroepiandrosterone (DHEA), DHEA-sulfate (DHEAS), free testosterone (FT) and total testosterone (TT). All of above noted laboratory tests were performed by routine commercial assays, as previously reported, with TT being evaluated with chromatography-tandem mass spectrometry [11].

We then assessed whether in Group 1 patients individual immune parameters or whole immune panels (i.e., thyroid antibodies, antiphospholipid antibodies, total immunoglobulins, etc.) statistically correlated with androgen levels.

As noted above, Group 2 patients, represented as subset 125 infertile women (mean age $38.9 \pm 5.4$ years), having undergone 169 IVF cycles within 2-4 weeks from last laboratory assessments (Table 1). Group 2 patients were used to assess whether immune parameters statistically correlated with embryo quality in IVF cycles.

Embryo quality was assessed in a total of 777 embryos. Each embryo is individually evaluated under the microscope early in the morning of day-3. Embryo assessments are performed at our center by counting blastomeres, assessing embryo shape, and determining the degree of fragmentation. Embryos were graded based on the following parameters: Excellent or Grade 5, reached 8 cells with $<5 \%$ fragmentation; Good or Grade 4 , reached $6-8$ cells with $\leq 5 \%$ fragmentation; Average or Grade 3, 6-8 cells with 5-20\% fragmentation; Poor or Grade 2, reached 4-8 cells with $20-40 \%$ fragmentation; Very poor or Grade 1, 4-8 cells, with $>40 \%$ fragmentation.

\section{Statistical analyses}

To investigate the effects androgen levels on immune system activation, generalized linear mixed-effects models (GLME) were used. GLME were also used to study the effects of immune system activation on embryo quality. The models were then controlled for age and repeated cycles in Group 2. Analyses were conducted using SAS 9.2. P-values $<0.05$ were considered statistically significant. All original data are available to investigators from CHR's electronic data depository by contacting David H. Barad, MD, MS at dbarad@thechr.com.

\section{Results}

Group-1 results

Patient characteristics for this group of infertility patients are summarized in Table 1. As the table demonstrates, their mean age was $38.6 \pm 5.4$ years. Their body mass index (BMI) was $24.0 \pm 4.9 \mathrm{~kg} / \mathrm{m}^{2}$.

It is also important to note that except for DHEAS and testosterone levels, none of the other patient characteristics differed between Groups 1 and 2. DHEA and testosterone levels increased in Group 2 patients because our center routinely supplements women with LFOR prior to IVF cycle start (see below).

Looking at the patients' individual levels of DHEA, DHEAS, TT, FT and TT/FT-ratios, none was individually associated with laboratory evidence of thyroid autoimmunity, presence of elevated antiphospholipid antibodies, antinuclear antibodies, anti-adrenal autoimmunity or anti-ovarian antibodies. TT/FT ratios were, however, statistically associated with gammopathies, either abnormally high or low total immunoglobulin levels in all isotypes ( $\mathrm{P}=0.026$; Table 2$)$.

\section{Group-2 results}

Even more interesting, however, were observations in Group 2, where the mean age was almost identical to Group-1 patients $(38.9 \pm 5.4$ years, Table 1$)$. Out of 777 embryos, 353 (45.4\%) were of good, 337 (43.4\%) were of fair and $87(11.2 \%)$ were of poor quality. Defining immune system activation as presence of one or more immune abnormalities, evidence for female immune system activation was associated with significantly better embryo quality than absence of such evidence $(P=0.008)$. This association, indeed, persisted even after adjustments for female age and repeat IVF cycles $(\mathrm{P}=0.006)$, strongly suggesting that mild immune system activation offers benefits in regard to embryo quality. The observed improvement in embryo quality was primarily the consequence of higher embryo grades $(3.7 \pm 0.05$ vs. $3.4 \pm 0.07 ; \mathrm{P}=0.002)$.

\section{Discussion}

Here presented data on random female infertility patients confirm the previously reported association between androgen levels and immune system activation [11], this time, however, even more specifically demonstrating that abnormalities in total immunoglobulin levels are statistically associated with TT/FT ratios.

\section{Table 2 Statistically significant associations}

\begin{tabular}{ll}
\hline Associations & Significance \\
\hline TT/FT with presence of gammopathies (lgG, IgM, IgA, IgE) & $P=0.026$ \\
Immune system activation* with embryo quality & $P=0.008$ \\
After adjustments for age and repeat cycles & $P=0.006$ \\
\hline
\end{tabular}

TT/FT, total/free testosterone ratio.

*As defined in Table 1. 
This is a potentially interesting new observation since $\mathrm{TT} / \mathrm{FT}$ ratios are to a large degree dependent on sex hormone binding globulin (SHBG), as defined by the Free Androgen Index $\left(F A I=100 \times \frac{\mathrm{TT}}{\mathrm{SHBG}}\right)$ [14]. While we, unfortunately, in this study did not have data on SHBG, these observations potentially link immunoglobulin concentrations via androgen levels to SHBG levels, as androgens are known to decrease SHBG [15,16], thus resulting in more of bioactive FT becoming available.

The association of TT/FT ratios and immunoglobulin abnormalities supports the previously reported association between mild immune system activation [11], normal ovarian function and normal androgen levels; while women with abnormally low ovarian function not only lack evidence of immune system activation but also demonstrate comparatively low androgen levels [10].

We, therefore, hypothesized about existence of a potential "immunologic" androgen production factor (APF), responsible for maintaining appropriate androgen levels for normal female fertility, which may be potentially increased in association with polycystic ovarian syndrome (PCOS) and decreased in women with LFOR ([11] and reviewed in [2]). Here presented data offer further support for such a hypothetical APF.

A possible association between immune system activation and embryo quality has never before ben reported. This observations in Group 2-women is, therefore, likely even more significant. It is not only statistically very robust but even held up after adjustment for age and number of earlier IVF cycles, suggesting that it is independent of female age and, at least to a degree, independent of severity of infertility.

Combined, these observations, therefore, suggest that a mild form of immune system activation may represent a highly important theme throughout all stages of successful reproduction, starting with egg/embryo quality, as here reported for the first time.

This concept is not necessarily new: For example, Jabbour et al. pointed out the importance of inflammatory pathways throughout reproductive physiology, from ovulation, to menstruation, implantation and onset of labor, all now increasingly perceived as inflammatory processes [17]. Here presented study suggests that creation of good embryo quality now also may have to be added as a process dependent on inflammatory pathways.

All of these observations also explain the close relationship and interplay between endocrine and immune systems, recently increasingly addressed in the literature [2,9].

How an activated immune system could beneficially affect egg and embryo quality remains to be established. Androgens, however, allow for a hypothesis: Recently reported animal models [18,19] and human clinical data ([20] reviewed in [21]), suggest that androgens are essential for early stages of follicle maturation. Improving egg/embryo quality via androgen supplementation in women with LFOR and with low androgen levels, therefore, appears to increase pregnancy chances [20], to reduce aneuploidy in embryos [22] and, therefore, miscarriage rates [23].

Androgens are, however, also strongly anti-inflammatory, and are, therefore, for that purpose clinically, for example, utilized in autoimmune disease [24]. The previously noted hypothetic immune system-derived APF with the ability to increases androgen levels, thus, potentially can create a feed-back loop by inducing anti-inflammatory androgens which, in turn, keep a low-level of inflammatory immune system activation in check.

In conclusion, here presented results add to an increasing volume of data pointing toward significant interaction between endocrine and immune systems in establishing reproductive success. Albertini recently described the ovaries as immunological "hot-spots" [25] based on the recognition that increasing numbers of fertility-associated genes in the ovary also have immunological functions. It increasingly appears that other endocrine organs may deserve a similar designation. Indeed, the brain and other endocrine organs like the adrenals, increasingly appear to represent locations where endocrine and immune systems almost merge into one immune/endocrine system (2). For successful reproduction the flawless function of this united system appears increasingly essential.

\section{Competing interests}

A.W., V.A. K., D.H.B and N.G. have in the past received research and travels support as well as speaker honoraria from pharmaceutical companies and/or medical device companies, none in any way related to here reported study except for the following: D.H.B. and N.G. receive patent royalties for a number of patents, claiming therapeutic benefits for androgen supplementation in women with low functional ovarian reserve. N.G. is shareholder of Fertility Nutraceuticals, LLC, a producer of fertility supplements, including DHEA. Both authors received travel support and speaker honoraria from Sun Pharmaceutical Industries, Ltd (India) for DHEA-related lectures. V.A.K. serves as consultant to the Centers for Disease Controls and Prevention in regards to ART outcome reporting.

\section{Authors' contributions}

AW. and NG. contributed equally to this study in design, data analysis and manuscript completion. EH. was a visiting fellow at the CHR during conduct of the study and significantly contributed to data accumulation. DHB. significantly contributed to study design, data analysis and manuscript preparation. VAK. contributed to study design and manuscript revisions. All other authors contributed to laboratory assessments. All authors approved the final manuscript.

\section{Author details}

${ }^{1}$ Center for Human Reproduction, New York, NY, USA. ${ }^{2}$ Department of Obstetrics and Gynecology, Medical University Vienna, Vienna, Austria. ${ }^{3}$ Centre Hospitalier de I'Université de Montréal, Montreal, Canada.

${ }^{4}$ Foundation for Reproductive Medicine, New York, NY, USA.

Received: 3 December 2014 Accepted: 15 March 2015

Published online: 11 April 2015

\section{References}

1. Hemberge M. Immune balance at the foeto-maternal interface as the fulcrum of reproductive success. J Reprod Immunol. 2013;97:36-42. 
2. Sen A, Kushnir VA, Barad DH, Gleicher N. Endocrine autoimmune diseases and female infertility. Nat Rev Endocrinol. 2014;10:37-50.

3. Higashi N, Niimi Y, Aoki M, Kawana S. Clinical features of antinuclear antibody-positive patients with atopic dermatitis. J Nippon Med Sch. 2009;76:300-7.

4. Mariz HA, Sato El, Barbosa SH, Rodrigues SH, Dellavance A, Andrade LE. Pattern of the antinuclear antibody-HEp-2 test is a critical parameter for discriminating antinuclear antibody-positive healthy individuals and patients with autoimmune rheumatic diseases. Arthritis Rheum. 2011;63:191-200.

5. Bruner BF, Guthridge JM, Lu R, Vidal G, Kelly JA, Robertson JM, et al. Comparison of autoantibody specificities between traditional and bead-based assays in al large, diverse collection of patients with systemic lupus erythematosus and family members. Arthritis Rheum. 2012;64:3677-86.

6. Turi A, Giannubilo SR, Zanconi S, Mascetti A, Tranquilli AL. Preconception steroid treatment in infertile women with antithyroid autoimmunity undergoing ovarian stimulation and intrauterine insemination: a double-blind, randomized, prospective cohort study. Clin Ther. 2010;32:2415-21.

7. Gleicher N, Weghofer A, Barad DH. Cutting edge assessment of the impact of autoimmunity on female reproductive success. J Autoimmun. 2012;38:174-80.

8. Gleicher N. Does the immune system induce labor? Lessons from preterm deliveries in women with autoimmune diseases. Clin Rev Allergy Immunol. 2010;39:194-206.

9. Gleicher N. Maternal autoimmunity and adverse pregnancy outcomes. J Autoimmun. 2014;50:83-6.

10. Gleicher N, Kim A, Weghofer A, Kushnir VA, Shohat-Tal A, Lazzaroni E, et al. Hypoandrogenism in association with diminished functional ovarian reserve. Hum Reprod. 2013;28:1048-91.

11. Gleicher N, Weghofer A, Kushnir VA, Shohat-Tal A, Lazzaroni E, Lee H-J, et al. Is androgen production in association with immune system activation potential evidence for existence of a functional adrenal/ovarian autoimmune system in women? Reprod Biol Endocrinol. 2013;11:58.

12. Grümmer R, Winterhager E. Blastocyst-mediated induction of endometrial connexins: an inflammatory response? J Reprod Immunol. 2011;90:9-13.

13. Bulla R, Bossi F, Tedesco F. The complement system ate the embryo implantation site: friend or foe? Front Immunol. 2012;19:55.

14. Ly LP, Handelsman DJ. Empirical estimation of free testosterone from testosterone and sex hormone-binding globulin immunoassay. Europ J Endocrinol. 2005;152:471-8.

15. Selby C. Sex hormone binding globulin: origin, function and clinical significance. Ann Clin Biochem. 1990;27(Pt6):532-41.

16. Nitsche R, Coelho JC, Freitas AC, Zeni Neto C, Martins E. Testosterone changes in patients with liver cirrhosis before and after orthotopic liver transplantation and its correlation with MELD. Arg Gastroenterol. 2014;51:59-63.

17. Jabbour HN, Sales KJ, Catalano RD, Norman JE. Inflammatory pathways in female reproductive health and disease. Reproduction. 2009;138:903-19.

18. Sen A, Hammes SR. Granulosa cell-specific androgen receptors are critical regulators of ovarian development and function. Molec Endocrinol. 2010;24:1393-403.

19. Narkwichean A, Jayaprakasan K, Maalouf WE, Hernandez-Medrano JH, Pincott-Allen C, Campbell BK. Effects of dehydroepiandrosterone on in vivo ovine follicular development. Hum Reprod. 2014;29:146-54.

20. Gleicher N, Kim A, Weghofer A, Shohat-Tal A, Lazzroni E, Lee H-J, et al. Starting and resulting testosterone levels after androgen supplementation determine at all ages in vitro fertilization (IVF) pregnancy rates in women with diminished ovarian reserve (DOR). J Assist Reprod Genet. 2013;30:49-62.

21. Lebbe M, Woodruff TK. Involvement of androgens in ovarian health and disease. Molec Human Reprod. 2013;19:828-37.

22. Gleicher N, Weghofer A, Barad DH. Dehydroepiandrosterone (DHEA) reduces embryo aneuploidy: direct evidence from preimplantation genetic screening (PGS). Reprod Biol Endocrinol. 2010;8:140

23. Gleicher N, Ryan E, Weghofer A, Blanco-Mejia S, Barad DH. Miscarriage rates after dehydroepiandrosterone (DHEA) supplementation in women with diminished ovarian reserve: a case control study. Reprod Biol Endocrinol. 2009;7:108.

24. Cuolo M, Seriolo B, Pizzorni C, Sulli A. The role of androgens in rheumatic diseases. IMAJ. 2001:3:743-8.

25. Albertini DF. Searching for answers to the riddle of ovarian aging. J Assist Reprod Genet. 2012;29:577-8.

\section{Submit your next manuscript to BioMed Central and take full advantage of:}

- Convenient online submission

- Thorough peer review

- No space constraints or color figure charges

- Immediate publication on acceptance

- Inclusion in PubMed, CAS, Scopus and Google Scholar

- Research which is freely available for redistribution

Submit your manuscript at www.biomedcentral.com/submit 